\title{
REDUCING DECOMPOSITIONS FOR STRICTLY CYCLIC OPERATORS
}

\author{
RICHARD BOULDIN
}

\begin{abstract}
If $T$ is a strictly cyclic operator on $H$ then $H$ has a direct sum decomposition $H_{1} \oplus H_{2}$ where $H_{1}$ and $H_{2}$ are invariant under $T$ if and only if the spectrum of $T$ is not connected. If $\lambda$ is a reducing eigenvalue for the strictly cyclic operator $T$ then the multiplicity of $\lambda$ is one and $\lambda$ is an isolated point of the spectrum of $T$.
\end{abstract}

1. Introduction. In [10] Nordgren showed that an injective weighted shift $S$ with square summable weights has the property that any densely defined closed operator commuting with $S$ is bounded. In [7] Lambert showed that the injective weighted shifts with the last property above are precisely those with strictly cyclic vectors. We say $e$ is a strictly cyclic vector for the operator $T$ on $H$ provided that $\mathscr{A}(T) e=\{A e: A \in \mathscr{A}(T)\}=H$ where $\mathscr{A}(T)$ is the smallest algebra of bounded operators which is closed in the operator norm and contains $T$; if $T$ has such a vector, then it is a strictly cyclic operator. The recent papers [2], [3], [8], [9] deduce various properties for strictly cyclic operators and strictly cyclic operator algebras.

In particular the existence of reducing subspaces is deduced in [2] and [9]. This is interesting since weighted shifts, which motivated the study of strictly cyclic operators, rarely have reducing subspaces (see Theorem 10 and Theorem 11 of [6]). So the existence of reducing subspaces is one measure of the difference between strictly cyclic operators and strictly cyclic weighted shifts.

In this paper we prove that a strictly cyclic operator has a nontrivial reducing decomposition if and only if the spectrum of the operator is not connected. Recall that the direct sum decomposition $H_{1} \oplus H_{2}=H$ is a reducing decomposition for the operator $T$ on $H$ provided that both $H_{1}$ and $H_{2}$ are invariant under $T$. We also show that any reducing eigenvalue for a strictly cyclic operator has multiplicity one and is an isolated point of the spectrum. With some results of [9] this shows that only a trivial class of strictly cyclic operators can have normal-like properties.

Received by the editors October 13, 1972.

AMS (MOS) subject classifications (1970). Primary 47B99; Secondary 47C05.

c) American Mathematical Society 1973 
2. Results. Our first theorem gives a basic new fact concerning convergence in the operator norm. Recall that a sequence of unit vectors, say $\left\{f_{n}\right\}$, is an approximate eigenvector corresponding to the approximate eigenvalue $\lambda$ provided that $\left\{\left\|(T-\lambda) f_{n}\right\|\right\}$ converges to zero. For the definition of residual spectrum see [4]. If $p(z)$ is the complex polynomial $\sum_{n=0}^{R} a_{n} z^{n}$ then $\bar{p}(z)$ denotes $\sum_{n=0}^{R} \bar{a}_{n} z^{n}$.

1. THEOREM. Let $\left\{f_{n}\right\}$ be an approximate eigenvector for $T$ corresponding to the approximate eigenvalue $\lambda$. If $A \in \mathscr{A}(T)$ and $\left\{p_{n}(z)\right\}$ is a sequence of complex polynomials such that $\left\{p_{n}(T)\right\}$ converges to $A$ in operator norm then $\left\{p_{n}(\lambda)\right\}$ converges to an approximate eigenvalue of $A$ with corresponding approximate eigenvector $\left\{f_{n}\right\}$. If $\alpha$ belongs to the residual spectrum of $T$ and $g$ is a normalized eigenvector for $T^{*}$ corresponding to $\bar{\alpha}$ then $\left\{p_{n}(\bar{\alpha})\right\}$ converges to an eigenvalue for $A^{*}$ with corresponding eigenvector $g$.

Proof. If $\lambda$ is an eigenvalue of $T$ and $f$ is a corresponding normalized eigenvector then the convergence of $\left\{p_{n}(T)\right\}$ to $A$ implies that $\left\{p_{n}(T) f=\right.$ $\left.p_{n}(\lambda) f\right\}$ converges to $A f$. This shows that $f$ is an eigenvector for $A$ with corresponding eigenvalue $\lim p_{n}(\lambda)$.

If $\lambda$ is an approximate eigenvalue of $T$ then we deduce the desired conclusion from Berberian's paper [1]. Using Berberian's notation, it is easy to see that $p_{n}(T)^{\circ}=p_{n}\left(T^{\circ}\right)$, where $p_{n}$ is a complex polynomial and $\left\{p_{n}(T)\right\}$ converges to $A$. Of course, $\lambda$ is an eigenvalue of $T^{\circ}$ with corresponding eigenvector any approximate eigenvector of $T$ corresponding to $\lambda$. Since Berberian shows that $\left\|B^{\circ}\right\|=\|B\|$, we see that the first paragraph above implies that $\left\{p_{n}(\lambda)\right\}$ converges to an eigenvalue $\mu$ of $A^{\circ}$ whose corresponding eigenvector is simply the approximate eigenvector of $T$ corresponding to $\lambda$. Thus $\mu$ is an approximate eigenvalue for $A$ with the corresponding approximate eigenvector being the aforementioned approximate eigenvector of $T$.

If $\alpha$ belongs to the residual spectrum of $T$ then $\bar{\alpha}$ is an eigenvalue of $T^{*}$ with some corresponding normalized eigenvector $g$. Since $\left\{p_{n}(T)\right\}$ converges to $A$ if and only if $\left\{\bar{p}_{n}\left(T^{*}\right)\right\}$ converges to $A^{*}$, it follows that $\left\{\bar{p}_{n}(\bar{\alpha})\right\}$ converges to an eigenvalue of $A^{*}$. Furthermore, $g$ is an eigenvector corresponding to $\lim \bar{p}_{n}(\bar{\alpha})$.

Because of its simplicity we state the following corollary which is immediate from the preceding theorem.

2. CoRollary. If $\left\{p_{n}(T)\right\}$ converges in the operator norm and $p_{n}(z)$ is a complex polynomial for each $n$ then for every $\lambda \in \sigma(T)$ the sequence $\left\{p_{n}(\lambda)\right\}$ converges to a point in the spectrum of $\lim p_{n}(T)$.

Our Example will make it clear that it would be unreasonable to expect all strictly cyclic operators to have only trivial reducing subspaces. 
Nevertheless, the next theorem shows that some strong assertions along these lines are true.

3. THEOREM. If $T$ is a strictly cyclic operator and $\sigma(T)$ is connected then $T$ has no nontrivial reducing decomposition.

Proof. We shall assume that the strictly cyclic operator $T$ has a nontrivial reducing decomposition $H=H_{1} \oplus H_{2}$ and we shall show that $\sigma(T)$ is not connected. We begin by showing that the projection of $H$ onto $H_{1}$ along $H_{2}$, which we call $P$, belongs to $\mathscr{A}(T)$. Let $e=e_{1}+e_{2}$ where $e$ is a strictly cyclic vector for $T$ and $e_{1} \in H_{1}, e_{2} \in H_{2}$. There is some $A_{1} \in \mathscr{A}(T)$ such that $A_{1} e=e_{1}$. It is routine to see that $e_{1}$ and $e_{2}$ are strictly cyclic vectors for $T / H_{1}$ and $T / H_{2}$, respectively. Thus for any $f \in H_{1}$ there is some $A_{2} \in \mathscr{A}(T)$ such that $A_{2} e=A_{2} e_{1}=f$ and since

$$
A_{1} A_{2} e_{1}=A_{1} A_{2} e=A_{2} A_{1} e=A_{2} e_{1}
$$

we see that $A_{1} / H_{1}$ is the identity. Since $A_{1}$ is zero on $H_{2}$, we see that $A_{1}=P$.

In order to deduce a contradiction we assume that $\sigma(T)$ is connected. Since $\sigma(T)=\sigma\left(T / H_{1}\right) \cup \sigma\left(T / H_{2}\right)$, it must be that the intersection of $\sigma\left(T / H_{1}\right)$ and $\sigma\left(T / H_{2}\right)$ is nonempty; take $\lambda$ in that intersection. Choose a sequence of complex polynomials so that $\left\{p_{n}(T)\right\}$ converges to $P$. Then $\left\{p_{n}\left(T / H_{2}\right)\right\}$ converges to $P / H_{2}$ and by Theorem $1\left\{p_{n}(\lambda)\right\}$ must converge to a point in the spectrum of $P / H_{2}$; thus $\lim p_{n}(\lambda)=0$. On the other, $\left\{p_{n}\left(T / H_{1}\right)\right\}$ converges to $P / H_{1}$ and so $\left\{p_{n}(\lambda)\right\}$ must converge to 1 . This contradiction proves that $\sigma\left(T / H_{1}\right)$ is disjoint from $\sigma\left(T / H_{2}\right)$ and so $\sigma(T)$ is not connected.

4. COROLlaRY. If $T$ is a strictly cyclic operator then $T$ has a nontrivial reducing decomposition if and only if $\sigma(T)$ is not connected.

Proof. This follows from the theorem and the well-known Riesz decomposition for an operator with separated spectrum. See p. 212 of [5], for example.

Since both unilateral weighted shifts and bilateral weighted shifts have connected spectrum (see Theorem 5 and Theorem 6 of [6]), it follows that a strictly cyclic weighted shift has no nontrivial reducing decomposition. It follows from Theorem 11 of [6] that if $S$ is a bilateral weighted shift with weights $\left\{a_{n}\right\}$ and if $\left\{\left|a_{n}\right|\right\}$ is periodic then $S$ is not strictly cyclic. The corollary gives some new information about unilateral weighted shifts which fail to be injective.

Before noting a further conclusion that follows from the proof of Theorem 3 we record the following basic fact.

5. LEMma. If $T$ has an eigenvalue $\lambda$ with multiplicity two or more then $T$ is not strictly cyclic. 
Proof. Let $\left\{f_{1}, f_{2}\right\}$ be an orthonormal set of $\lambda$-eigenvectors and let $e$ be a strictly cyclic vector for $T$. Then $e$ has a unique representation

$$
e=c_{1} f_{1}+c_{2} f_{2}+f_{3}+f_{4}
$$

where $c_{1}, c_{2}$ are scalars, $f_{3}$ is a vector from $\operatorname{ker}(T-\lambda)$ orthogonal to $\left\{f_{1}, f_{2}\right\}$ and $f_{4}$ is orthogonal to $\operatorname{ker}(T-\lambda)$. For any complex polynomial $p$ we have

$$
p(T) e=p(\lambda)\left(c_{1} f_{1}+c_{2} f_{2}+f_{3}\right)+p(T) f_{4}
$$

and we can choose two sequences of complex polynomials, say $\left\{p_{n}\right\},\left\{q_{n}\right\}$, such that $\left\{p_{n}(T) e\right\}$ converges to $f_{1}$ while $\left\{q_{n}(T) e\right\}$ converges to $f_{2}$. Thus $\left\{p_{n}(\lambda)\left(c_{1} f_{1}+c_{2} f_{2}+f_{3}\right)+p_{n}(T) f_{4}\right\}$ converges to $f_{1}$ which implies that $c_{2}=0$, $f_{3}=0,\left\{p_{n}(T) f_{4}\right\}$ converges to 0 and $\left\{p_{n}(\lambda) c_{1}\right\}$ converges to 1 . Because $\left\{q_{n}(\lambda)\left(c_{1} f_{1}+c_{2} f_{2}+f_{3}\right)+q_{n}(T) f_{4}\right\}$ converges to $f_{2}$, it must be that $c_{1}=0$, $f_{3}=0,\left\{q_{n}(T) f_{4}\right\}$ converges to 0 and $\left\{q_{n}(\lambda) c_{2}\right\}$ converges to 1 . These conclusions are obviously inconsistent and the lemma is proved.

5. THEOREM. If $\lambda$ is an eigenvalue for the strictly cyclic operator $T$ and $\operatorname{ker}(T-\lambda)$ reduces $T$ then $\lambda$ is an isolated eigenvalue with multiplicity one.

Proof. The conclusion about the multiplicity is immediate from Lemma 5. In the proof of Theorem 3 if one takes $H_{1}=\operatorname{ker}(T-\lambda)$ and $H_{2}=(\operatorname{ker}(T-\lambda))^{\perp}$ then one concludes that $\{\lambda\}=\sigma\left(T / H_{1}\right)$ is disjoint from $\sigma\left(T / H_{2}\right)$ and thus $\lambda$ is an isolated point of $\sigma(T)$.

Using the above theorem it is possible to sharpen Theorem 2 of [2].

6. Corollary. If $T$ is a strictly cyclic hyponormal operator then $T$ is a finite direct sum of one dimensional operators with each operator corresponding to a different scalar.

Proof. By Lambert's Theorem 3.3 in [9], there is a subspace $M$ which reduces $T$ to a normal operator with finite spectrum and $\left\|T / M^{\perp}\right\|>$ $r\left(T / M^{\perp}\right)$ if $M^{\perp}$ is nontrivial. Since $T / M^{\perp}$ must be hyponormal, it follows that $M=H$ and this corollary follows from Theorem 5 .

Finally we want to demonstrate that the trivial normal operators mentioned in Corollary 6 are not typical of the operators described in Theorem 5.

7. EXAMPLE. Let $T_{0}$ be a strictly cyclic contraction operator defined on $H_{0}$ with strictly cyclic vector $f$. Let $H_{1}$ be a one dimensional Hilbert space and set $T=T_{0} \oplus \mu I$ on $H_{0} \oplus H_{1}$ where $|\mu|>1$. Then $T$ is strictly cyclic.

Proof. Let $X=\sigma\left(T_{0}\right) \cup\{\mu\}$ and note that the function $f(z)$ defined to be zero on $\sigma\left(T_{0}\right)$ and one on $\{\mu\}$ is continuous on $X$ and holomorphic 
on the interior of $X$. By Mergelyan's theorem there is a sequence of polynomials, say $\left\{p_{n}(z)\right\}$, such that $\left\{p_{n}(z)\right\}$ converges to $f(z)$ uniformly on $X$. Because $\{z:|z| \leqq 1\}$ is a spectral set for $T_{0}$ we see that $\left\{p_{n}\left(T_{0}\right)\right\}$ must converge to the zero operator. Since $\left\{p_{n}(\mu)\right\}$ converges to one, the sequence $\left\{p_{n}(T)\right\}$ must converge in operator norm to the orthogonal projection of $H$ onto $H_{1}$; call that projection $P$. Since $P \in \mathscr{A}(T)$ we see that

$$
\mathscr{A}(T)(g+e) \supset \mathscr{A}(T) P(g+e)=H_{1}
$$

where $g$ belongs to $H_{0}$ and $e$ is a unit vector in $H_{1}$.

Now we show that $\left(T_{0}-\mu\right) f$ is a strictly cyclic vector for $T_{0}$. The Neumann series $\left(I-\mu^{-1} T_{0}\right)^{-1}=\sum_{k=0}^{\infty}\left(\mu^{-1} T_{0}\right)^{k}$ converges in the operator norm and so $\left(T_{0}-\mu\right)^{-1}$ belongs to $\mathscr{A}\left(T_{0}\right)$. Clearly

$$
\mathscr{A}\left(T_{0}\right)\left(T_{0}-\mu\right) f=\mathscr{A}\left(T_{0}\right) f=H_{0}
$$

and so $\left(T_{0}-\mu\right) f$ is a strictly cyclic vector for $T_{0}$. If $e$ is a unit vector in $H_{1}$ then

$$
\mathscr{A}(T)(T-\mu)(f+e)=\mathscr{A}\left(T_{0}\right)\left(T_{0}-\mu\right) f=H_{0} .
$$

Since $\mathscr{A}(T)(f+e)$ is a linear manifold in $H$ containing both $H_{0}$ and $H_{1}$, it follows that $\mathscr{A}(T)(f+e)=H$.

\section{BIBLIOGRAPHY}

1. S. K. Berberian, Approximate proper vectors, Proc. Amer. Math. Soc. 13 (1962), 111-114. MR 24 \#A3516.

2. R. Bolstein and W. Wogen, Subnormal operators in strictly cyclic operator algebras (preprint).

3. M. Embry, Strictly cyclic operator algebras on a Banach space, Pacific J. Math. (to appear).

4. P. R. Halmos, A Hilbert space problem book, Van Nostrand, Princeton, N.J., 1967. MR $34 \# 8178$.

5. T. Kato, Perturbation theory for linear operators, Die Grundlehren der math. Wissenschaften, Band 132, Springer-Verlag, New York, 1966. MR 34 \#3324.

6. R. L. Kelley, Weighted shifts on Hilbert space, Dissertation, University of Michigan, Ann Arbor, Mich., 1966.

7. A. Lambert, Strictly cyclic wrighted shifts, Proc. Amer. Math. Soc. 29 (1971), 331-336. MR 43 \#970.

8. - Strictly cyclic operator algebras, Pacific J. Math. 39 (1971), 717-726.

9. - Spectral properties of strictly cyclic operator algebras (preprint).

10. E. Nordgren, Closed operators commuting with a weighted shift, Proc. Amer. Math. Soc. 24 (1970), 424-428. MR 41 \#2435.

Department of Mathematics, University of Georgia, Athens, Georgia 30601 